\title{
Inappropriate Hepatic Cholesterol Synthesis Expands the Cellular Pool of Sterol Available for Recruitment by Bile Acids in the Rat
}

\author{
Lyman E. Bilhartz, David K. Spady, and John M. Dietschy \\ Department of Medicine, University of Texas Southwestern Medical Center, Dallas, Texas 75235-8887
}

\begin{abstract}
These studies test the hypothesis that a major determinant of excessive biliary cholesterol secretion is a level of hepatic sterol synthesis that is inappropriately high relative to the needs of the liver cell for preserving cholesterol balance. Biliary cholesterol secretion was measured in vivo in two models after loading the hepatocyte with sterol by two different mechanisms. In the first model, cholesterol was delivered physiologically to the liver in chylomicron remnants. This resulted in a sixfold increase in cholesteryl ester content and marked suppression of cholesterol synthesis, but biliary cholesterol secretion remained essentially constant. In the second model, 3-hydroxy-3-methyl-glutaryl CoA reductase levels in the liver were markedly increased by chronic mevinolin (lovastatin) administration. Withdrawal of the inhibitor resulted in a sudden fivefold increase in the rate of sterol synthesis in the liver of the experimental animals that was inappropriately high for cellular needs. This excessive synthesis, in turn, was accompanied by a fivefold increase in the cholesteryl ester content, enrichment of microsomal membranes with cholesterol and, most importantly, by a threefold increase in the rate of biliary sterol secretion. As the rate of sterol synthesis gradually returned to normal over $48 \mathrm{~h}$, the cholesterol ester content, the lipid composition of the microsomal membranes, and rate of cholesterol secretion into bile also returned to baseline values. These results further support the concept of functional compartmentalization of cholesterol in the hepatocyte. Derangements that cause an inappropriately high rate of sterol synthesis in the endoplasmic reticulum may lead to an expansion of that pool of cholesterol that is recruitable by bile acids and, hence, to greater saturation of the bile.
\end{abstract}

\section{Introduction}

The most common cause of gallstone disease in Western populations is either a relative or absolute increase in cholesterol secretion by the liver, yet there is little understanding of the basic mechanism(s) responsible for this phenomenon $(1,2)$. Cholesterol is an important component of the liver cell and serves several functionally different roles. It may, for example, act as a structural component in certain cell membranes, as a substrate for enzymes such as acyl-CoA:cholesterol acyl trans-

Address correspondence to Dr. Bilhartz, Department of Medicine, University of Texas Southwestern Medical Center, 5323 Harry Hines Blvd., Dallas, TX 75235-8887. 1989.

Received for publication 15 April 1988 and in revised form 3 June

J. Clin. Invest.

(c) The American Society for Clinical Investigation, Inc.

$0021-9738 / 89 / 10 / 1181 / 07 \$ 2.00$

Volume 84, October 1989, 1181-1187 ferase $(\mathrm{ACAT})^{1}$ and cholesterol $7 \alpha$-hydroxylase and as a putative regulator of the synthesis of 3-hydroxy-3-methylglutaryl (HMG) CoA reductase and the LDL receptor (3-6). Furthermore, a small fraction of this cellular cholesterol is available for recruitment into the bile by bile acids, and this pathway represents one of the two major mechanisms for the excretion of the sterol molecule from the body (3). The cholesterol that is required for these varied roles comes from only three sources: it may be (a) absorbed from the diet, $(b)$ synthesized in the extrahepatic tissues and delivered to the liver, or $(c)$ synthesized within the liver itself. Under physiological circumstances changes in the rates of delivery of sterol from the intestine and extrahepatic organs to the liver is compensated for by reciprocal changes in the rate of hepatic cholesterol synthesis. In this manner the net balance of cholesterol across the liver cell remains essentially unchanged. Furthermore, under these physiological conditions, the pool of sterol within the hepatocyte that is recruitable by bile acids also appears to remain essentially constant $(7,8)$. Hence, the rate of biliary cholesterol secretion, and, therefore, the degree of biliary cholesterol saturation, remains relatively constant under circumstances where there may be marked changes in dietary cholesterol intake or de novo cholesterol synthesis.

However, recent data from a number of different sources now suggest that this tightly regulated system may become metabolically deranged in such a way that bile acids passing through the liver are able to recruit significantly greater amounts of cholesterol and so produce bile that is more saturated with respect to sterol. For example, hamsters that are deficient in essential fatty acids have a rate of hepatic cholesterol synthesis that is elevated 15-fold and rates of biliary cholesterol secretion that are increased over sevenfold (9). Similarly, when rats are treated with pregnenolone-16 $\alpha$-carbonitrile and an agent that blocks cholesterol absorption, hepatic sterol synthesis and biliary cholesterol secretion are again markedly elevated (10). In both of these situations suppression of hepatic cholesterol synthesis leads to suppression of biliary cholesterol output. Thus, in contrast to the normal physiological situation where biliary cholesterol is independent of hepatic synthesis, in these pathological states the pool of sterol that is recruitable by bile acids appears to depend directly upon the rate of cholesterol synthesis in the hepatocyte. A similar abnormal state may exist in the obese human where a correlation has also been described between the rate of cholesterol synthesis and the degree of saturation of the bile $(1,11)$.

Unfortunately, these models are complex since the treatments may induce other changes in liver metabolism in addition to the excessive rate of cholesterol synthesis. Thus, the purpose of this study was to determine directly if inappropri-

1. Abbreviations used in this paper: ACAT, acyl-CoA:cholesterol acyl transferase; HMG, 3-hydroxy-3-methylglutaryl. 
ately high rates of cholesterol synthesis expand the pool of cholesterol available for bile acid recruitment under circumstances where the flux of bile acids across the liver was held constant. These studies took advantage of the observation that chronic administration of the HMG-CoA reductase inhibitor, mevinolin, leads to synthesis of excessive amounts of this rate limiting enzyme in the cholesterol biosynthetic pathway (12, 13). In such animals, the rate of hepatic cholesterol synthesis measured in vivo with $\left[{ }^{3} \mathrm{H}\right] \mathrm{H}_{2} \mathrm{O}$ is normal since the excessive amounts of HMG-CoA reductase are partially inhibited by the presence of the mevinolin (unpublished observations; the generic drug name for mevinolin is lovastatin). However, when the inhibitor is abruptly removed from the diet, this excess reductase becomes manifest, and hepatic cholesterol synthesis markedly increases in a manner that is transiently inappropriate for cellular needs. Thus, in this study we quantitated the effect of introducing cholesterol into the liver either physiologically in chylomicron remnants or unphysiologically by inducing unregulated sterol synthesis. The effect of these two manipulations on the recruitment of cholesterol into bile and on other metabolic pathways was then measured.

\section{Methods}

Materials. Mevinolin was provided by Merck Sharp \& Dohme Research Laboratories (Rahway, NJ). Glycocholic acid (sodium salt) was obtained from Calbiochem-Behring Corp. (LaJolla, CA), $\left[{ }^{3} \mathrm{H}\right] \mathrm{H}_{2} \mathrm{O}$ from ICN (Irvine, CA), and [cholesteryl-4- ${ }^{14} \mathrm{C}$ ]cholesteryl oleate, [24- $\left.{ }^{14} \mathrm{C}\right]$-taurocholate, and $\left[1,2-{ }^{3} \mathrm{H}(\mathrm{N})\right]$ cholesterol from New England Nuclear Products (Boston, MA).

Experimental model. Female, Sprague Dawley-derived rats (Sasco, Inc., Omaha, NE), originally purchased in the $125-150 \mathrm{~g}$ weight range were kept in a room with alternating periods of light (1500-0300) and darkness (0300-1500), allowed water ad lib. and fed rodent diet (Wayne Laboratory Animal Diets, Chicago, IL) for at least 2 wk before being used. Chylomicrons were obtained from donor rats fitted with intestinal lymphatic cannulae and infused intragastrically with an eggyolk emulsion (14).

In the first group of experiments, exogenous cholesterol in the form of chylomicrons was administered as a bolus intravenous injection at the beginning of the dark phase of the light cycle. The dose of the chylomicron bolus was adjusted so that each animal received $80 \mathrm{mg}$ of chylomicron-cholesterol per $\mathrm{kg}$ of body weight. Control animals received a saline injection. Separate groups of animals were killed 0,6 , 12,24 , and $48 \mathrm{~h}$ after the chylomicron bolus for determination of the hepatic cholesteryl ester content. The in vivo rate of hepatic cholesterol synthesis was measured at the same time points in a parallel group of animals using $\left[{ }^{3} \mathrm{H}\right] \mathrm{H}_{2} \mathrm{O}$ (15). A final group of animals was fitted with femoral vein and bile duct catheters and the endogenous bile acid pool was drained. Bile flow was maintained by an exogenous infusion of glycocholate $(8.8 \mathrm{mM})$ in saline at a rate adjusted so that each animal maintained a constant bile acid flux across the liver of $100 \mu \mathrm{mol} / \mathrm{h}$ per $\mathrm{kg}$ body weight. Bile was collected in hourly aliquots and assayed for the concentrations of bile acids, phospholipids, and cholesterol.

A second group of experiments was carried out in a manner similar to that described above except that instead of the chylomicron infusion, the rats were pretreated for 1 wk with a diet containing $1,000 \mathrm{mg}$ of mevinolin per $\mathrm{kg}$ of ground diet. At the beginning of the dark phase (designated time $0 \mathrm{~h}$ ) the diet containing mevinolin was removed. The rate of in vivo hepatic cholesterol synthesis was measured at the time points described above, and in a separate group of animals bile was collected during an exogenous infusion of glycocholate as described above. In a third group of animals, the endogenous bile acid pool was labeled by instilling about $100,000 \mathrm{dpm}$ of ${ }^{14} \mathrm{C}$-labeled taurocholate intragastrically $24 \mathrm{~h}$ before placement of a bile fistula. Bile was col- lected in hourly aliquots and assayed for radioactivity to determine the time course for drainage of the endogenous bile acid pool. A final group of animals was killed at $0,6,12,24$, and $48 \mathrm{~h}$ after the mevinolin was withdrawn. Aliquots of whole liver were taken for determination of cholesterol ester content, and microsomal membranes were isolated from the remaining liver as described (16), and the membranes were assayed for their protein, phospholipid, and cholesterol content.

Hepatic cholesterol content and biliary lipid analysis. Hepatic cholesteryl esters were separated from free cholesterol using silicic acid/ celite column chromatography (17), saponified, and then quantified by gas-liquid chromatography. The biliary concentration of bile acids was measured by reversed-phase high performance liquid chromatography (Waters Associates, Medford, MA) with a differential refractometer as the detector. The mobile phase contained $65 \%$ methanol/35\% $0.6 \mathrm{M}$ acetate buffer, $\mathrm{pH} 4.5$, pumped at $1.2 \mathrm{ml} / \mathrm{min}$ through a $3.9-\mathrm{mm}$ $\times 30-\mathrm{cm} \mu$ Bondapak $\mathrm{C}_{18}$ Column. The content of cholesterol in bile, plasma, and in microsomal membranes was measured by gas-liquid chromatography and the phospholipid content of bile and membranes were determined as described (18). The biliary lipid concentrations were multiplied by the bile flow rates to obtain the lipid secretion rates. The units are $\mathrm{nmol}$ of lipid secreted per $\mathrm{h}$ per $\mathrm{g}$ wet weight of liver.

In vivo rate of hepatic cholesterol synthesis. To determine the in vivo rate of hepatic cholesterol synthesis the animals were injected intravenously with $25 \mathrm{mCi}$ of $\left[{ }^{3} \mathrm{H}\right] \mathrm{H}_{2} \mathrm{O}$ and killed $1 \mathrm{~h}$ later. Blood was drawn to determine the specific activity of the plasma water and aliquots of liver were weighed and saponified with alcoholic $\mathrm{KOH}$. Hepatic sterols were isolated by precipitation with digitonin after which the digitonides were split with pyridine and assayed for radioactivity $(15,19)$. The absolute mass of cholesterol synthesized during the 1-h test period was calculated using experimental data showing that $24 \mathrm{H}$ atoms from water are incorporated into each cholesterol molecule under the conditions of these experiments (20). The units are nanomoles of cholesterol synthesized per hour per gram wet weight of liver.

Statistical analysis. In the tables and figures an asterisk indicates that the experimental value is significantly different from the appropriate control valve using a Student's $t$ test $(P<0.05)$. All data represent mean values \pm 1 SEM.

\section{Results}

In control animals under basal conditions, each gram of liver contained $4,740 \pm 180 \mathrm{nmol}$ of free cholesterol and $520 \pm 60$ $\mathrm{nmol}$ of esterified cholesterol. Over the course of $1 \mathrm{~h}$ that same gram of liver synthesized $160 \pm 20 \mathrm{nmol}$ of cholesterol and secreted $40 \pm 5 \mathrm{nmol}$ of cholesterol into bile.

The first study was designed to illustrate how these normal parameters of hepatic sterol metabolism respond to a sudden, physiological increase in the load of cholesterol presented to the liver. As shown in Fig. 1, these basal conditions were perturbed by administration of a bolus of chylomicrons at time 0 . The control group of animals received only a saline injection, and neither the cholesteryl ester content, the rate of cholesterol synthesis, nor the rate of biliary cholesterol secretion changed over the course of the experiment. In the chylomicron-infused group, the lipoproteins were rapidly cleared from the plasma, and within hours cholesteryl esters began to accumulate in the liver (Fig. $1 A$ ) and hepatic cholesterol synthesis was suppressed (Fig. $1 B$ ). The effect of the chylomicron bolus was maximal $12 \mathrm{~h}$ after injection, and both the ester content and the rate of cholesterol synthesis then returned to normal by 48 h. In contrast, despite loading the hepatocyte with cholesterol the rate of biliary cholesterol secretion was not significantly different from those values found in the control animals (Fig. $1 C)$. 

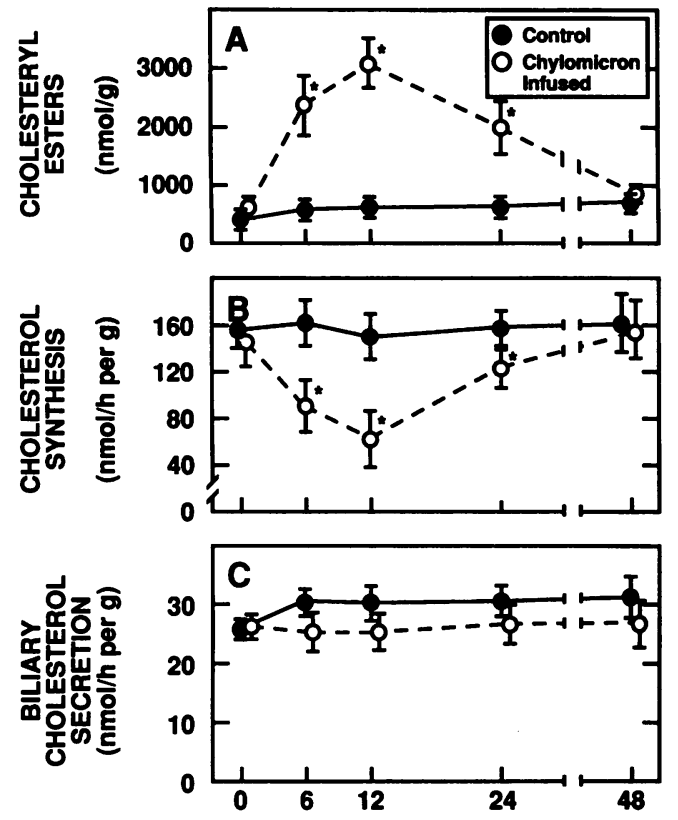

TIME AFTER MEVINOLIN WITHDRAWAL (h)

Figure 1. The response of hepatic cholesteryl ester levels and cholesterol synthesis, and biliary cholesterol secretion to a physiological load of cholesterol. At time 0 each animal received intravenously either saline alone or a bolus of chylomicrons containing $80 \mathrm{mg}$ of cholesterol per kg body weight. Groups of these animals were then used to determine the rates of cholesterol synthesis and biliary cholesterol secretion in vivo, as well as the concentration of cholesteryl esters in the liver. Each point represents the mean \pm 1 SEM for six animals. ${ }^{*} P<0.05$.

In the second set of studies, the liver was also suddenly presented with an excess load of sterol. In this case, however, the load came from the inappropriate, unphysiological overproduction of cholesterol within the endoplasmic reticulum of the liver cell. To accomplish this, animals were fed a diet containing the cholesterol synthesis inhibitor, mevinolin, for $1 \mathrm{wk}$ which results in a marked increase in the content of HMG CoA reductase, the rate-limiting enzyme in the cholesterol biosynthetic pathway. Since the activity of the inhibitor is lost more rapidly than the activity of the enzyme after withdrawal of the drug, there is a sudden and inappropriate overproduction of cholesterol in the liver of the experimental animals.

Fig. 2 shows the time course required for drainage of the endogenous bile acid pool in the control animals and the mevinolin-withdrawn animals. As shown, in both groups, 95\% of the endogenous bile acid pool was drained between 12 and $18 \mathrm{~h}$ after the fistula was established. Thus, mevinolin had no direct effect on the enterohepatic circulation of bile acids; moreover, the quantity of endogenous bile acids secreted during this same period approximates the rate of bile acid synthesis.

The major findings of this study are shown in Fig. 3. The rate of cholesterol synthesis in vivo increased nearly sixfold, from $167 \pm 24$ to $911 \pm 35 \mathrm{nmol} / \mathrm{h}$ per $\mathrm{g}, 12 \mathrm{~h}$ after withdrawing the mevinolin (Fig. 3 B). Much of this newly synthesized sterol was apparently esterified since the cholesteryl ester content of the liver also increased (Fig. $3 A$ ). In marked contrast to the first experiment, however, this inappropriate increase in the

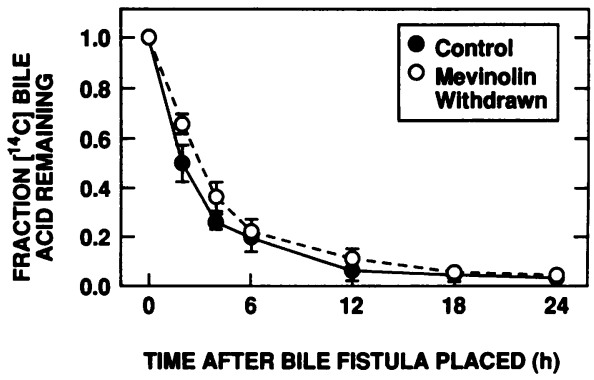

Figure 2. Time course for drainage of the endogenous bile acid pool after placement of a bile fistula. The animals were administered $\sim 100,000 \mathrm{dpm}$ of $\left[{ }^{14} \mathrm{C}\right]$ taurocholic acid in saline intragastrically 24 $\mathrm{h}$ before placement of a bile fistula. The fractional secretory rate of the labeled taurocholate is plotted as a function of time. Each point represents the mean \pm 1 SEM for six animals; there were no significant differences between the two groups in the time required to drain the endogenous bile acid pool.

cholesterol load to the liver resulted in a marked increase in the rate of cholesterol secretion into bile. At $12 \mathrm{~h}$, for example, the experimental group was secreting $263 \pm 42 \mathrm{nmol} / \mathrm{h}$ per $\mathrm{g}$ of cholesterol compared with $102 \pm 13 \mathrm{nmol} / \mathrm{h}$ per $\mathrm{g}$ in the control group. It should be emphasized that the rate of bile acid secretion was carefully maintained at a constant value in both groups of animals so that the excessive cholesterol secretion seen in the treated group could not be ascribed to an increased bile acid flux across the liver.
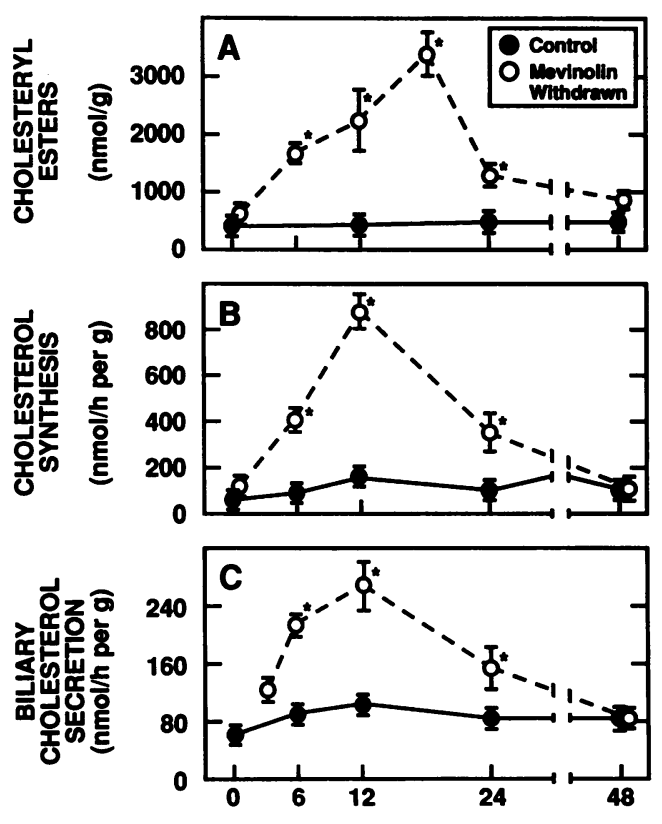

TIME AFTER MEVINOLIN WITHDRAWAL (h)

Figure 3. The response of hepatic cholesteryl ester levels and cholesterol synthesis, and biliary cholesterol secretion to an inappropriate overproduction of cholesterol by the liver. These animals were pretreated for $1 \mathrm{wk}$ with either a controlled diet or a diet supplemented with mevinolin ( $1,000 \mathrm{mg}$ per $\mathrm{kg}$ of diet). At time 0 the mevinolin treatment was discontinued, and groups of animals were used to make the same measurements shown in Fig. 1. Each point represents the mean \pm 1 SEM for 10 animals. ${ }^{*} P<0.05$. 
Table I shows the biliary secretion of bile acids, phospholipids, and cholesterol 12, 24, and $48 \mathrm{~h}$ after withdrawal of the inhibitor during infusion of the exogenous glycocholic acid. During the critical 12-h time point, when cholesterol synthesis was at its maximum, the total bile acid flux was virtually identical in the two groups, yet phospholipid and cholesterol secretion were both significantly elevated. Moreover, the composition of the bile acid flux driving lipid secretion was the same in the two groups (87\% glycocholate, $5 \%$ tauromuricholate, $5 \%$ taurocholate, and 3\% taurochenodeoxycholate), and, thus, changes in cholesterol recruitment could not be ascribed to either quantitative or qualitative (21) alterations in the bile acid flux that was driving lipid secretion.

In Fig. 4 the molar ratios of the three biliary lipids are plotted as a function of the time after withdrawal from mevinolin. In the control group, the molar ratios of the three biliary lipids remained essentially constant during the $48 \mathrm{~h}$ of bile acid infusion. In contrast, the exaggerated sterol synthesis brought about by mevinolin withdrawal gave rise to marked pertubations in the lipid ratios. Each mole of bile acid traversing the liver recruited more than twice as much cholesterol at the point of maximal disequilibrium when compared with the control group (Fig. $4 A$ ). The ratio of cholesterol to bile acid remained significantly elevated for $24 \mathrm{~h}$ and only returned to normal when the rate of cholesterol synthesis returned to normal. The ratio of cholesterol to phospholipid demonstrated a similar pattern (Fig. $4 C$ ). Finally, and somewhat surprisingly, phospholipid secretion was also significantly increased during this interval (Fig. 4 B).

In an effort to determine if expansion of the recruitable cholesterol pool was associated with alterations in the lipid content of any subcellular membrane compartment, hepatic microsomal membranes were isolated from control animals and from animals $0,8,16,24$, and $48 \mathrm{~h}$ after withdrawal from mevinolin. The results of this experiment are shown in Fig. 5. When expressed as nanomoles of lipid per milligram of microsomal membrane protein, the phospholipid content of these membranes remained constant throughout the period of withdrawal of mevinolin. In contrast, the cholesterol content of these membranes increased from a baseline value in the control group of $90 \pm 4 \mathrm{nmol}$ per mg protein to $158 \pm 11 \mathrm{nmol}$ per mg protein. Moreover, this selective enrichment of this membrane compartment with cholesterol corresponded in time to the period of inappropriate cholesterol synthesis and to the acute expansion of the recruitable cholesterol pool.

\section{Discussion}

In any species, including human beings, two of the major determinants of the rate of cholesterol movement into bile are the absolute amounts of bile acid traversing the liver $(22,23)$ and the chemical structure of the particular bile acids contained within the enterohepatic circulation. Thus, changes in either the absolute flux or the relative hydrophobicity of the bile acid pool alters the amount of sterol that is recruited from the liver (21). However, several lines of evidence now suggest that changes in these two parameters are insufficient in most cases to account for the excessive amount of cholesterol that appears in the bile in pathological states in man and in several animal models. Thus, we and other investigators (24-28) have suggested that the principle abnormality causing the production of bile that is supersaturated with cholesterol may be an expansion of an intracellular pool of sterol that is recruitable by bile acids, rather than a quantitative or qualitative change in the bile acid pool.

In the regulated, physiological state, the rate of hepatic cholesterol synthesis fully compensates for changes in the rate of sterol delivery to the liver from the intestine and other extra-hepatic organs and in the rate of sterol degradation (3, 29). A change in the net balance of cholesterol across the liver is associated with an appropriate, and reciprocal, change in the rate of hepatic sterol synthesis (Fig. $1, A$ and $B$ ). Under these circumstances, the pool of hepatic cholesterol that is recruitable by bile acids apparently remains constant (Fig. $1 C$ ) although the contribution of newly synthesized cholesterol to this pool may vary severalfold $(7,30)$. While the anatomical location of this recruitable pool is uncertain, it clearly is functionally distinct since the specific activity of biliary cholesterol is consistently greater than the specific activity of sterol in the liver as a whole after administration of $\left[{ }^{3} \mathrm{H}\right] \mathrm{H}_{2} \mathrm{O}$ to the animal (30).

Table I. Biliary Lipid Secretion Induced by a Controlled Bile Acid Flux after Withdrawal from Mevinolin

\begin{tabular}{|c|c|c|c|c|c|c|}
\hline & \multirow{2}{*}{$\begin{array}{c}\text { Time after mevinolin } \\
\text { withdrawal }\end{array}$} & \multirow[b]{2}{*}{ Bile flow } & \multicolumn{2}{|c|}{ Bile acid secretion } & \multirow[b]{2}{*}{ Phospholipid secretion } & \multirow[b]{2}{*}{ Cholesterol secretion } \\
\hline & & & Endogenous & Exogenous & & \\
\hline & $h$ & $\mathrm{ml} / \mathrm{h}$ per $\mathrm{kg}$ & \multicolumn{2}{|c|}{$\mu \mathrm{mol} / \mathrm{h}$ per $\mathrm{kg}$} & $\mu \mathrm{mol} / \mathrm{h}$ per $\mathrm{kg}$ & $\mu \mathrm{mol} / \mathrm{h}$ per $\mathrm{kg}$ \\
\hline Control & 12 & $3.61 \pm 0.23$ & $14 \pm 1$ & $87 \pm 3$ & $23.7 \pm 1.5$ & $2.10 \pm 0.27$ \\
\hline Mevinolin & 12 & $4.26 \pm 0.47$ & $13 \pm 2$ & $87 \pm 2$ & $31.8 \pm 2.2^{*}$ & $4.31 \pm 0.64^{*}$ \\
\hline Control & 24 & $3.64 \pm 0.12$ & $11 \pm 2$ & $90 \pm 1$ & $22.1 \pm 1.0$ & $2.02 \pm 0.08$ \\
\hline Mevinolin & 24 & $4.47 \pm 0.35$ & $8 \pm 1$ & $93 \pm 6$ & $27.9 \pm 2.4$ & $2.62 \pm 0.29$ \\
\hline Control & 48 & $3.78 \pm 0.36$ & $11 \pm 3$ & $91 \pm 3$ & $22.4 \pm 2.5$ & $2.00 \pm 0.17$ \\
\hline Mevinolin & 48 & $4.82 \pm 0.62$ & $8 \pm 2$ & $93 \pm 5$ & $26.7 \pm 3.1$ & $1.84 \pm 0.22$ \\
\hline
\end{tabular}

Female rats were fed a control diet or a diet supplemented with mevinolin for $1 \mathrm{wk}$. A bile fistula was placed, and after the endogenous bile acid pool had been drained, glycocholic acid $(8.8 \mathrm{mM})$ was infused intravenously at a rate calculated to maintain a total bile acid flux across the liver of $100 \mu \mathrm{mol} / \mathrm{h}$ per $\mathrm{kg}$ body weight. Endogenous bile acids were separated from the exogenous glycocholate by HPLC and quantified. $* P<0.05$. 


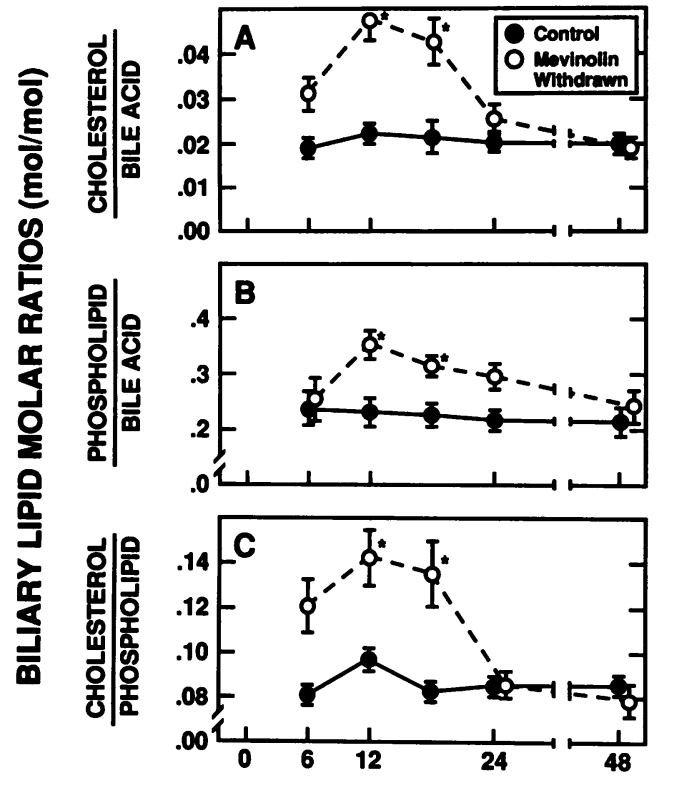

TIME AFTER MEVINOLIN WITHDRAWAL (h)

Figure 4. Molar ratios of the three biliary lipids during mevinolin-induced, inappropriate cholesterol synthesis in the liver. In these studies the bile acid flux across the liver was kept constant at $100 \mu \mathrm{mol} / \mathrm{h}$ per $\mathrm{kg}$ body weight. Each point represents the mean \pm 1 SEM for 10 animals. ${ }^{*} P<0.05$.

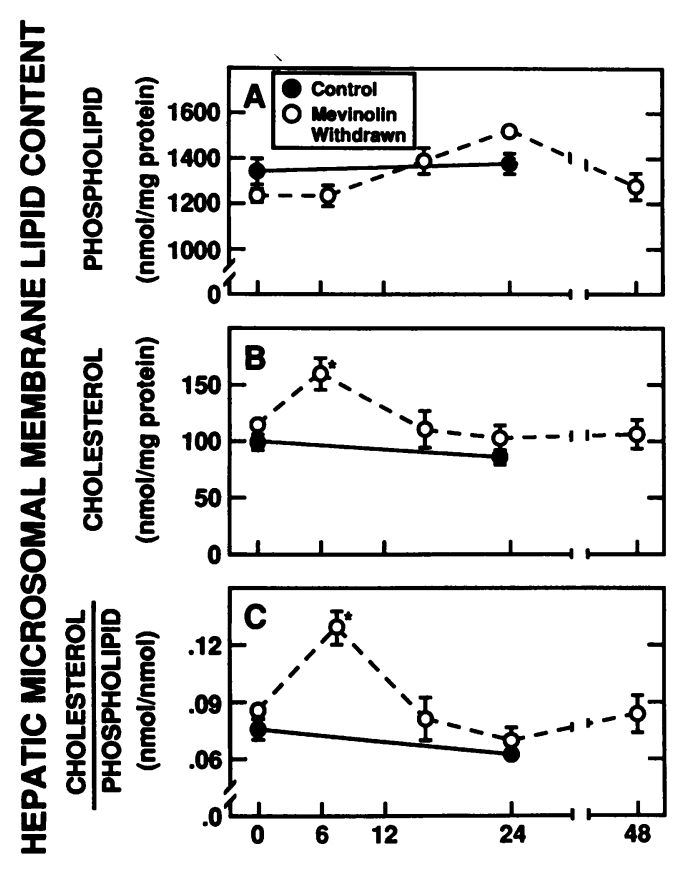

TIME AFTER MEVINOLIN WITHDRAWAL (h)

Figure 5. Hepatic microsomal membrane lipid content as a function of time after withdrawal from mevinolin. 20 mevinolin fed animals were divided into five equal groups and killed $0,8,16,24$, and $48 \mathrm{~h}$ after withdrawal from the inhibitor. The hepatic microsomal membranes were isolated as described and assayed for their protein and lipid content. $8 \mathrm{~h}$ after withdrawal from mevinolin, the cholesterol content of the membranes and the ratio of cholesterol to phospholipid was significantly elevated. Each point represents the mean \pm 1 SEM for four animals. ${ }^{*} P<0.05$.
In contrast to this situation in the normal animal (7) and in humans (31), there are several pathological situations where the recruitable hepatic cholesterol pool is no longer independent of the rate of cholesterol synthesis. For example, hamsters fed a fat-free diet manifest a 3- and 16-fold increase, respectively, in whole-body and liver cholesterol synthesis rates, and these changes were associated with a sevenfold increase in the output of cholesterol in bile (9). Similarly, treatment of the rat with pregnenolone- $16 \alpha$-carbonitrile increased the rate of both hepatic cholesterol synthesis and biliary cholesterol secretion (10). Furthermore, in both of these models, when the rate of hepatic cholesterol synthesis was varied by altering the amount of cholesterol reaching the liver from the intestine, the rate of hepatic cholesterol secretion varied directly with the rate of hepatic synthesis. Thus, paradoxically, cholesterol feeding suppressed both hepatic sterol synthesis and biliary cholesterol secretion (10) and has even been reported to prevent cholesterol gallstone formation (32). Finally, as seen in these two animal models, in the obese human there is also a direct correlation between whole-body cholesterol synthesis and biliary cholesterol secretion (33). Taken together, these results suggest that in these pathological states, the rate of hepatic cholesterol synthesis is no longer tightly coupled to the needs of the liver cell, and, thus, under these abnormal circumstances, cholesterol is synthesized in excessive amounts and secreted into the bile. Other possible explanations for these observations include metabolic abnormalities that might have been induced by these treatments or by the presence of obesity in the human subjects.

The current studies, however, provide direct support for the hypothesis that a rate of sterol synthesis that is inappropriate for cellular needs may expand the cholesterol pool that can be recruited by bile acids and thereby result in excessive cholesterol secretion into bile. The animals treated with mevinolin for 1 wk exhibited no discernible metabolic abnormalities in terms of sterol metabolism at the beginning of the experiment. Rates of cholesterol synthesis in the whole animal and liver, in particular, were normal, as was the rate of cholesterol secretion into bile. The free cholesterol content of microsomal membranes, the cholesterol ester content of the hepatocyte, as well as the plasma cholesterol concentration were all within the normal range at the outset of the experiments. After withdrawal of the mevinolin, however, the expanded pool of HMG-CoA reductase became immediately manifest, and there was inappropriate overproduction of cholesterol (Fig. 3). This acutely expanded pool of newly synthesized sterol transiently enriched the hepatic microsomal membrane fraction. ACAT, located in this same membrane compartment, was able to esterify a portion of this excess free cholesterol, but a significant fraction was recruited by the bile acid flux and secreted into bile. As the excess HMG-CoA reductase activity gradually declined, the rate of sterol synthesis returned to normal along with the free cholesterol content of the microsomal membrane fraction and the rate of cholesterol secretion into bile. Notably, when a similar mass of cholesterol was delivered to the hepatocyte via the receptor-mediated endocytosis of a chylomycron remnant, no expansion of the recruitable cholesterol pool occurred.

In this rat model, mevinolin, an inhibitor of cholesterol synthesis, was used to paradoxically increase the rate of cholesterol synthesis in the hepatocyte. The results of this study should not be taken to suggest that chronic use of mevinolin in 
human subjects would necessarily result in a similar phenomenon. Indeed, other investigators (34) have observed that a different HMG-CoA reductase inhibitor actually lowered the degree of cholesterol saturation of human gallbladder bile. The seeming contradiction between the current study in rats and the observations made in humans could probably be reconciled if it were known whether the reductase inhibitor administered to humans suppressed or stimulated (or both) hepatic cholesterol synthesis. Unfortunately, data are sparse on the overall effect of HMG-CoA reductase inhibitors on the in vivo rate of cholesterol synthesis in humans (35) and certainly, nothing is known about the hour to hour rate of hepatic cholesterol synthesis in humans. In any event, as a model, these results clearly support the hypothesis that one of the major causes of excessive biliary cholesterol secretion, and presumably gallstone formation, may be a metabolic abnormality (induced, for example, by obesity) that leads to an inappropriately high rate of cholesterol synthesis in the liver, an expanded pool of recruitable sterol and, hence, excessive entrainment of cholesterol into the bile by an essentially normal flux of bile acid across the liver. Such a model further supports the idea that the defect in cholesterol gallstone disease is within the realm of intracellular cholesterol trafficking rather than in the enterohepatic circulation of bile acids.

\section{Acknowledgments}

We thank Alfred Alberts of Merck Sharp \& Dohme Research Laboratories for kindly supplying the mevinolin, Luis Rodriquez and Jacob Bilhartz for their technical assistance, and Bettye Culwell for administrative assistance.

This work was supported by U. S. Public Health Service grants AM-19329, HL-09610, and RR-07175 and grants from the Moss Heart Fund and the Ruby D. Hexter Estate. Dr. Bilhartz is the recipient of an American Gastroenterological Association/Industry Research Scholar Award.

\section{References}

1. Bennion, L. J., and S. M. Grundy. 1978. Risk factors for the development of cholelithiasis in man. N. Engl. J. Med. 299:11611227.

2. Bilhartz, L. E. 1988. Cholesterol gallstone disease: the current status of nonsurgical therapy. Am. J. Med. Sci. 295:45-56.

3. Turley, S. D., and J. M. Dietschy. 1988. The metabolism and excretion of cholesterol by the liver. In The Liver: Biology and Pathology. I. M. Arias, W. B. Jacoby, H. Popper, D. Schachter, and D. A. Schafritz, editors. Raven Press, New York. 617-641.

4. Sampson, W. J., Suffolk, R. A., Bowers, P., Houghton, J. D., Botham, K. M., and Suckling, K. E. 1987. The role of acyl-CoA: cholesterol acyltransferase in the metabolism of free cholesterol to cholesteryl esters or bile acids in primary cultures of rat hepatocytes. Biochim. Biophys. Acta 920:1-8.

5. Brown, M. S., and J. L. Goldstein. 1986. A receptor-mediated pathway for cholesterol homeostasis. Science (Wash. DC). 232:34-47.

6. Spady, D. K., and J. M. Dietschy. 1988. Interaction of dietary cholesterol and triglycerides in the regulation of hepatic low density lipoprotein transport in the hamster. J. Clin. Invest. 81:300-309.

7. Turley, S. D., and J. M. Dietschy. 1979. Regulation of biliary cholesterol output in the rat: dissociation from the rate of hepatic cholesterol synthesis, the size of the hepatic cholesteryl ester pool, and the hepatic uptake of chylomicron cholesterol. J. Lipid Res. 20:923934.

8. Spady, D. K., S. D. Turley, and J. M. Dietschy. 1983. Dissocia- tion of hepatic cholesterol synthesis from hepatic low-density lipoprotein uptake and biliary cholesterol saturation in female and male hamsters of different ages. Biochim. Biophys. Acta. 753:381-392.

9. Turley, S. D., D. K. Spady, and J. M. Dietschy. 1983. Alteration of the degree of biliary cholesterol saturation in the hamster and rat by manipulation of the pools of preformed and newly synthesized cholesterol. Gastroenterology. 84:253-264.

10. Turley, S. D., and J. M. Dietschy. 1984. Modulation of the stimulatory effect of pregnenolone-16 $\alpha$-carbonitrile on biliary cholesterol output in the rat by manipulation of the rate of hepatic cholesterol synthesis. Gastroenterology. 87:284-292.

11. Bennion, L. J., and S. M. Grundy. 1976. Effects of obesity and caloric intake on biliary lipid metabolism in man. J. Clin. Invest. 57:473-477.

12. Chin, D. J., K. L. Luskey, R. G. W. Anderson, J. R. Faust, J. L. Goldstein, and M. S. Brown. 1982. Appearance of crystalloid endoplasmic reticulum in compactin-resistant Chinese hamster cells with a 500-fold increase in 3-hydroxy-3-methylglutaryl-coenzyme A reductase. Proc. Natl. Acad. Sci. USA. 79:1185-1189.

13. Roitelman, J., and I. Shechter. 1986. Altered kinetic properties of rat liver 3-hydroxy-3-methylglutaryl coenzyme A reductase following dietary manipulations. J. Biol. Chem. 261:5061-5066.

14. Nervi, F. O., and J. M. Dietschy. 1978. The mechanisms of and the interrelationship between bile acid and chylomicron-mediated regulation of hepatic cholesterol synthesis in the liver of the rat. J. Clin. Invest. 68:895-909.

15. Jeske, D. J., and J. M. Dietschy. 1980. Regulation of rates of cholesterol synthesis in vivo in the liver and carcass of the rat measured using $\left[{ }^{3} \mathrm{H}\right]$ water. J. Lipid Res. 21:364-376.

16. Brown, M. S., J. L. Goldstein, and J. M. Dietschy. 1979. Active and inactive forms of 3-hydroxy-3-methylglutaryl coenzyme A reductase in the liver of the rat. J. Biol. Chem. 254:5144-5149.

17. Andersen, J. M., and J. M. Dietschy. 1978. Relative importance of high and low density lipoproteins in the regulation of cholesterol synthesis in the adrenal gland, ovary, and testis of the rat. J. Biol. Chem. 253:9024-9032.

18. Bartlett, G. R. 1959. Phosphorus assay in column chromatography. J. Biol. Chem. 234:466-468.

19. Turley, S. D., J. M. Andersen, and J. M. Dietschy. 1981. Rates of sterol synthesis and uptake in the major organs of the rat in vivo. $J$. Lipid Res. 22:551-569.

20. Dietschy, J. M., and D. K. Spady. 1984. Measurement of rates of cholesterol synthesis using tritiated water. J. Lipid Res. 25:14691476.

21. Bilhartz, L. E., and J. M. Dietschy. 1988. Bile salt hydrophobicity influences cholesterol recruitment from rat liver in vivo when cholesterol synthesis and lipoprotein uptake are constant. Gastroenterology. 95:771-779.

22. Hofmann, A. F., and A. Roda. 1984. Physicochemical properties of bile acids and their relationship to biological properties: an overview of the problem. J. Lipid Res. 25:1477-1489.

23. Carey, M. C., and M. J. Cahalane. 1988. Enterohepatic circulation. In The Liver: Biology and Pathology. 2nd edition. I. M. Arias, W. B. Jacoby, H. Popper, D. Schacter, and D. A. Schafritz, editors. Raven Press, New York. 573-616.

24. Bilhartz, L. E., and J. M. Dietschy. 1985. Lipoprotein metabolism, hepatic cholesterol pools, and cholesterol gallstone formation. In Recent Advances In Bile Acid Research. L. Barbara, R. H. Dowling, A. F. Hofmann, and E. Roda, editors. Raven Press, New York. 165-171.

25. Stone, B. G., S. K. Erickson, W. Y. Craig, and A. D. Cooper. 1985. Regulation of rat biliary cholesterol secretion by agents that alter intrahepatic cholesterol metabolism. J. Clin. Invest. 76:1773-1781.

26. Robins, S. J., J. M. Fasulo, M. A. Collins, and G. M. Patton. 1985. Evidence for separate pathways of transport of newly synthesized and preformed cholesterol into bile. J. Biol. Chem. 260:65116513. 
27. Kern, F., Jr., and G. T. Everson. 1987. Contraceptive steroids increase cholesterol in bile: mechanisms of action. J. Lipid Res. 28:828-839.

28. Nervi, F., I. Marinovic, A. Rigotti, and N. Ulloa. 1988. Regulation of biliary cholesterol secretion: functional relationship between the canalicular and sinusoidal cholesterol secretory pathways in the rat. J. Clin. Invest. 82:1818-1825.

29. Spady, D. K., E. F. Stange, L. E. Bilhartz, and J. M. Dietschy. 1986. Bile acids regulate hepatic low density lipoprotein receptor activity in the hamster by altering cholesterol flux across the liver. Proc. Natl. Acad. Sci. USA. 83:1916-1920.

30. Turley, S. D., and J. M. Dietschy. 1981. The contribution of newly synthesized cholesterol to biliary cholesterol in the rat. J. Biol. Chem. 256:2438-2446.

31. Maton, P. N., A. Reuben, and R. H. Dowling. 1982. Relationship between hepatic cholesterol synthesis and biliary cholesterol se- cretion in man: hepatic cholesterol synthesis is not a major regulator of biliary lipid secretion. Clin. Sci. (Lond.). 62:515-519.

32. Dam, H., I. Prange, and E. Søndergaard. 1974. Alimentary production of gallstones in hamsters. Z. Ernährungswiss. 13:208-236.

33. Grundy, S. M., H. Y. I. Mok, and K. von Bergmann. 1976. Regulation of biliary cholesterol secretion in man. In The Liver: Quantitative Aspects of Structure and Function. R. Preisig, J. Bercher, G. Paumgartner, editors. Editio Cantor, Aulendorf, Germany. 393-403.

34. Duane, W. C., D. B. Hunninghake, M. L. Freeman, P. A. Pooler, L. A. Schlasner, and R. L. Gebhard. 1988. Simvastatin, a competitive inhibitor of HMG-CoA reductase, lowers cholesterol saturation index of gallbladder bile. Hepatology. 8:1147-1150.

35. Grundy, S. M., and D. W. Bilheimer. 1984. Inhibition of 3-hydroxy-3-methylglutaryl-CoA reductase by mevinolin in familial hypercholesterolemia heterozygotes: effects on cholesterol balance. Proc. Natl. Acad. Sci. USA. 81:2538-2542. 